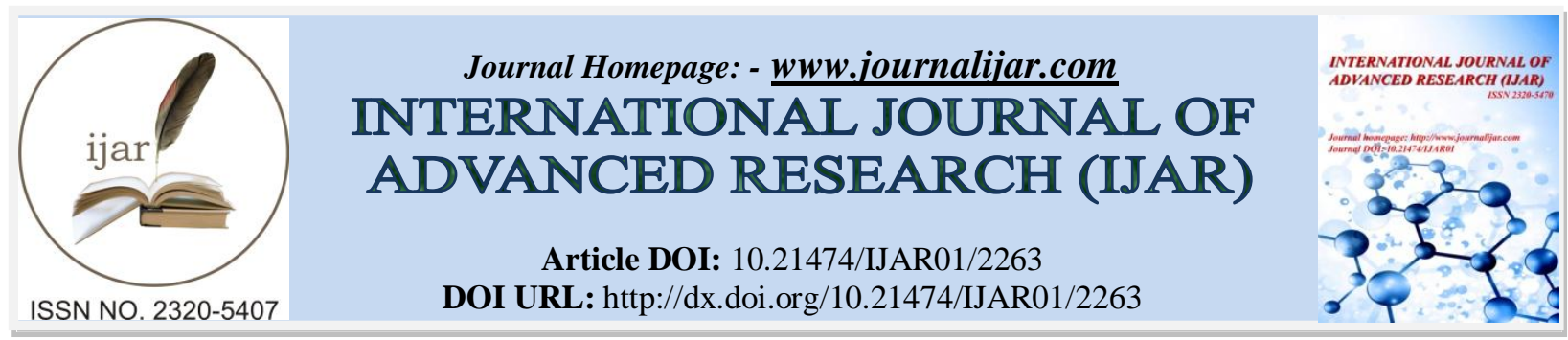

RESEARCH ARTICLE

\title{
IMAGING TECHNIQUES FOR THE IDENTIFICATION OF MIDDLE EAR CHOLESTEATOMA: A REVIEW.
}

Kirti Somani ${ }^{1}$, Nupur Tewari ${ }^{1}$, Khandakar Faridar Rahman ${ }^{2}$ and Saurabh Mukherjee ${ }^{2}$.

1. M.tech. Scholar, Department of Computer Science, AIM \& ACT, Banasthali University, Rajasthan, India.

2. Associate professo, Department of Computer Science, AIM \& ACT, Banasthali University, Rajasthan, India.

\section{Manuscript Info}

Manuscript History

Received: 29 September 2016

Final Accepted: 30 October 2016

Published: November 2016

Key words:-

Cholesteatoma, Mastoidectomy, Tympanoplasty, Computed Tomography (CT), Echo-planar Diffusion Weighted Magnetic Resonance Imaging. Non echo-planar Diffusion Weighted

Magnetic Resonance Imaging.

\section{Abstract}

The purpose of this study is to recognize the various imaging techniques that have been introduced in order to identify and detect the middle ear disease, Cholesteatoma. In the existing studies many different techniques such as computed tomography, high resolution computed tomography, multi-detector computed tomography, multislice computed tomography, diffusion weighted echo-planar magnetic resonance imaging, and diffusion weighted non-echo planar magnetic resonance imaging have been proposed. This paper consists of the case studies that have been undertaken by the researchers along with the advantages and limitations of the techniques being used.

Copy Right, IJAR, 2016,. All rights reserved.

\section{Introduction:-}

Cholesteatoma is a type of skin cyst or pouches that shed layers of old skins and is located in the middle ear mastoid bone in the skull. It is usually caused due to repeated infection or because of the poor Eustachian tube function. The Eustachian tube conveys air from the back of the nose into the middle ear to help equalize ear pressure. When the Eustachian tube is not working well, the negative pressure builds up and pulls part of the eardrum inwards, creating a vacuum in the ear. This creates a cyst that is filled with old skin and other waste material.

\section{Cholesteatoma of the middle ear and temporal bone are divided as follows:-}

$>$ Congenital cholesteatoma accounting only for $2 \%$.

$>$ Acquired cholesteatoma accounting for $98 \%$

$\rightarrow$ primary acquired cholesteatoma(no history of chronic otomastoiditis)

$\rightarrow$ secondary acquired cholesteatoma(vast majority)

- Pars flaccida (attic) cholesteatoma

- Pars tensa (sinus) cholesteatoma

$>$ External ear canal cholesteatoma

$>$ Mural cholesteatoma

For the initial detection of cholesteatoma various imaging techniques are used. Computed Tomography (CT) is earliest detection technique used, also High Resolution Computed Tomography (HRCT) is used. Apart from Computed Tomography, Magnetic Resonance Imaging (MRI) is also used. There are many variations in magnetic

Corresponding Author:- Kirti Somani.

Address:- M.tech. Scholar, Department of Computer Science, AIM \& ACT, Banasthali University, 
resonance imaging such as: echo spin diffusion weighted MRI, non-echo spin diffusion weighted MRI, late post T1 gadolinium diffusion weighted MRI.

Cholesteatoma very often continues to grow if not removed. Although surgery is rarely urgent, but once detected, the only solution to cholesteatoma is middle ear surgery. There are mainly two types of surgeries for cholesteatoma:

$\rightarrow$ Mastoidectomy (surgery performed when the infection or cholesteatoma has grown into the mastoid).

$\rightarrow$ Tympanoplasty (surgery performed to repair the eardrum and hearing mechanism).

\section{Analysis:- \\ Mihael Ries et al.:-}

This proposed paper represents one case report of Bilateral Congenital Cholesteatoma (CC) in middle ear as an extremely rare disease. A 3 year old boy was diagnosed with right sided acute otitis media (AOM), accompanied by discrete facial nerve palsy on the side of the involved ear. For a complete surgical removal of cholesteatoma and facial nerve decompression a canal wall down (CWD) technique was performed.

\section{Critical Remark:-}

Treatment of $\mathrm{CC}$ requires early surgery and long-term follow-up. This case confirmed the fact that the otomicroscopy is insufficient for the detection of middle ear cholesteatoma, especially when there is no eardrum perforation or nearby bony effect.

\section{Filippo Di Lella etal:-}

The main aim of this proposed paper was to describe the clinical features, pathogenesis and surgical results in a series of adult patients suffering from middle ear cholesteatoma behind an intact tympanic membrane(ITMC).A total of 27 cases were identified, out of which 10 cases showed a history of recurrent episodes of otorrhoea, 4 cases showed a history of previous middle ear surgery for non-cholesteatomatous chronic otitis media, and in the remaining 2 cases, a cranial trauma was considered.

\section{Critical Remark:-}

The chief complaint of the patients was hearing loss, other symptoms evolved were facial paralysis ( 1 case), dizziness ( 2 cases), ear fullness ( 3 cases), otalgia (5 cases), and tinnitus (13 cases).

\section{Andrea Elefante et al:-}

This proposed paper's main objective was to evaluate the advantage of Multi-Shot Turbo Spin Echo(MSh TSE) DWI (thickness $3 \mathrm{~mm}$ ) with shot imaging time(about $2 \mathrm{~min}$ ) over Single-Shot Echo-Planar(SSh EPI) DWI with longer imaging time(about $8 \mathrm{~min}$ ) for the diagnosis of cholesteatoma. A clinical finding over 32 patients with suspicion of unilateral cholesteatoma was performed with MRI (1.5T) SSh EPI and MSh TSE, 16 patients were suspected of having primary cholesteatoma and 16 having recurrent diseases.

\section{Critical Remark:-}

The findings suggested that-1) MSh TSE showed higher diagnostic accuracy and lower negative predictive value (NVP) compared to conventional SSh EPI. 2) MSh TES DWI has higher sensitivity, specificity for detection of cholesteatoma and lower probability of misdiagnosis. 3) MSh TES DWI is useful in guiding less experienced observers to the diagnosis. 4) MSh TES DWI can provide great support in daily clinical practice compared to SSh EPI. The main problems of this technique are susceptibility artifacts at the skull base, motion artifacts due to cardiac movements/ blood vessels pulsation and longer imaging time.

\section{Robert Nash et al:-}

This proposed paper's main objective was to represent the operative findings for 158 cases of which 54 were children's, with the aim to access the diagnostic performance of non-eco planar DWI with Half-Fourier single-shot turbo-spin echo. Secondly, to compare the diagnostic performance of DWI used for detection of post operative cholesteatoma in children's with that in the adult patient group. Performing DWMRI in children's is a challenging task as children's may have difficulty in tolerating the examination, therefore to overcome this sedation may be needed. Another imaging technique used for the detection of post operative cholesteatoma was delayed contrast MRI. In some cases a group of selected patients were present in whom DWMRI imaging is replacing second look surgery. This study supports DWMRI as a valuable tool in detecting post-operative cholesteatoma in children's and lends support in a similar way to adults. 


\title{
Critical Remark:-
}

$>$ It was acknowledged that a small lesion less than 2-4 mm may not be detected using DWMRI technique.

$>$ In DW-MRI, movement during the scan causes misregistration and degradation of the acquired image and therefore do not produce an accurate result.

$>$ There was no statistical significant difference in the specificity (which was greater than $85 \%$ ), sensitivity (which was greater than $90 \%$ ), positive or negative predictive values between children and adults.

\begin{abstract}
Ahmed Abdel Razek et al:-
Researchers' main aim was to establish a computed tomography staging of middle ear cholesteatoma, and assessing its impact on the selection of the surgical procedure. CT is a valuable tool and plays an important role in the assessment of patient with cholesteatoma of middle ear. The CT staging classified cholesteatoma according to its location within the tympanic cavity (T), extent of mastoid involvement (M), and associated complications (C). Case study was conducted on 61 patients. The overall sensitivity of CT staging of cholesteatoma compared to surgery was $88 \%$ with excellent agreement and correlation between CT findings and intra operative findings.
\end{abstract}

\section{Critical Remarks:-}

$\mathrm{CT}$ is valuable for the initial imaging for the cholesteatoma but differentiation of cholesteatoma and scar tissue is impossible using $\mathrm{CT}$ and requires non echo planar diffusion weighted MR imaging.

\section{S Velthuis et al.:-}

The main aim of the researchers' were to determine the value of non echo planar, diffusion weighted magnetic resonance imaging for detecting of residual and recurrent middle ear cholesteatoma after combined approach Tympanoplasty. The method used was that magnetic resonance imaging findings of cholesteatoma after primary surgery were compared with intra-operative findings at 'second-look' surgery or with clinical follow-up findings.

Statistical analysis were done and the usefulness of non echo planar, diffusion weighted MRI for the detection of residual/recurrent cholesteatoma after cholesteatoma surgery was described in terms of the means of positive and negative predictive values and sensitivity and specificity. 38 patients case studies were taken, out of which no falsepositive findings were there but there were four cases of false negative findings.

\section{Critical Remarks:-}

The result of using non echo planar diffusion weighted MRI had shown promising result. It helped to reduce the number of unnecessary second-look procedure performed. 2) However, if non echo planar, diffusion weighted MRI was used instead of second look surgery, strict clinical follow up and regular MRI studies are compulsory as false negative results may occur.

\section{Sang Hyuk Ahn et al.:-}

In this proposed paper the authors' have presented usefulness of Computed Tomography Hounsfield Unit (HU) for the diagnosis of congenital cholesteatoma. A total of 43 patients underwent surgery out of which 21 patients were confirmed to have congenital cholesteatoma, and 22 patients were confirmed to have otitis media (OM) by operation. The mean HU value for congenital cholesteatoma was 37.36+-6.11(range 27.5-52.5) and 76.09+8.74(range 58.5-96) in OM group, and HU cut-off value was 55.5.

\section{Critical Remark:-}

According to the recent study Hounsfield measurements proved to be an aid for the diagnosis of cholesteatoma. The two type of cholesteatoma congenital and acquired differ in their location, clinical history and status of tympanic membrane but they are not distinguishable histologically, therefore we hypothesized that the HU measurement in the temporal bone CT would be useful for the diagnosis of congenital cholesteatoma.

\section{Ana Flavia Assis de Avila et al.:-}

The paper proposed by researchers contains the imaging evaluation of middle ear cholesteatoma. Researchers had discussed various imaging techniques used in the detection of cholesteatoma, ranging from multi-slice computed tomography (MSCT), delayed post gadolinium T1 weighted sequences, and diffusion weighted sequences or a combination of both techniques. 


\section{Critical Remarks:-}

$>$ Multi-slice computed tomography was considered the initial imaging method for the detection of middle ear cholesteatoma and demonstrate sensitivity ranging between $70-90 \%$ but cannot define if the tissue is inflammatory, fibrotic or cholesteatoma.

$>$ Whereas in diffusion weighted magnetic resonance imaging there are two variations, echo planar imaging and non-echo planar imaging. Non echo planar imaging has thinner thickness and was less pre-disposed to susceptibility artifacts than echo planar sequences.

\section{Maged B Naguib:-}

This proposed paper is case report describing the surgical management of petrous bone cholesteatoma, a rare form of epidermoid cyst affecting the petrous part of the temporal bone. In this study 5 patients with petrous bone cholesteatoma are presented, all underwent high resolution CT of temporal bone for diagnosis. Based on the CT topographic location, the petrous bone cholesteatoma was classified into - supralabyrinthine (2 cases), infralabyrinthine ( 2 cases) and infralabyrinthine apical (1 case).

\section{Critical Remark:-}

$>$ CT scan was carried out not only for the detection of petrous bone cholesteatoma bust also for the exact localization of the lesion.

$>$ The common symptom found in all these patients was gradual hearing loss, and in few facial nerve paralysis.

$>$ All the cases were surgically managed by performing a subtotal petrosectomy.

\section{C.Eduardo Corrales and Nikolas H.Belvins:-}

This proposed paper's main objective was to diagnose cholesteatoma, with emphasis on high-resolution computed tomography (HRCT) and diffusion-weighted MRI (DW-MRI). DW-MRI imaging has proven to be a reliable method by showing high sensitivity and specificity in detecting recidivistic cholesteatoma down to $3 \mathrm{~mm}$ in size, and allows radiologic differentiation between cholesteatoma and other soft tissue .HRCT can identify cholesteatoma with specificity between $80-90 \%$.

\section{Critical Remark:-}

HRCT limits its ability to differentiate between soft tissue in the temporal bone to other fluids and tissues commonly seen in the chronics otitis media. HRCT has proven to be a useful roadmap for surgery to help guide the surgeons.

\section{Lauren L.Levy et.al:-}

In this proposed paper the authors' have presented the use of Optical-Imaging with contrast enhanced High Resolution Micro-Endoscopy (HRME) for the detection of ectopic keratin production in Middle Ear Cholesteatoma. Optical-imaging technology provides non-invasive visualization of tissue epithelium in real time and endoscopic technique using HRME allows for the spatial resolution of individual nuclei and cellular structures. An Ex-vivo imaging and in-vivo of histopathologically is also compared.

\section{Critical Remark:-}

$>$ The HRME for imaging head and neck squamous cell, obstructed visualization of nuclei and was a limitation in distinguishing benign from cancerous mucosa.

$>$ Ex-vivo imaging was performed on fresh specimens immediately following surgical resection.

$>$ in-vivo images showed higher contrast than Ex-vivo images of fresh or frozen/thawed tissue.

$>$ Temperature difference was found in the imaging features of two.

$>$ Ex-vivo demonstrated the feasibility of proflavine-enhanced HRME to distinguish cholesteatoma from uninvolved middle ear mucosa, in-vivo are required to test the hypothesis that intra-operative real-time imaging can enhance surgical removal.

\section{Dalia Monir Fahmy et al.:-}

The purpose of this study was to determine the role of propeller diffusion weighted MR imaging combined with conventional MR imaging for the detection of residual cholesteatoma in patients who have undergone middle ear surgery.

Twenty cases were taken and variations were found in results. Two had lesion smaller than $5 \mathrm{~mm}$ and could not be evaluated, one was misdiagnosed and the rest were nine granulation tissue and eight cases of residual cholesteatoma. 
The method study here showed sensitivity of $80 \%$, specificity of $90 \%$, and positive predictive value of $89 \%$ and negative predictive value of $82 \%$.

\section{Critical Remarks: -}

$>$ though the study showed a good statistic of the technique, it had its own disadvantages. Its main disadvantage is low spatial resolution, which results from the fact that the periphery of $k$-space is more sparsely filled than its central region.

$>$ Also this technique take long duration, increased scanning time when compared to DWI-EPI.

\section{K. Yamashita et al.:-}

The main purpose of the study was to evaluate the advantage of Multi-shot Echo Planar Imaging (MS-EPI) for the diagnosis of middle ear cholesteatoma by comparing it with single-shot echo planar imaging (SS-EPI).

Case studies of 29 patients were taken, all of them suspected with acquired cholesteatoma. Each patient underwent MRI examination including both MS-EPI and SS-EPI. Results showed that both techniques are excellent. MS-EPI was associated with higher sensitivity and accuracy than SS-EPI, while both methods showed $100 \%$ specificity.

\section{Critical Remarks: -}

$>$ Results and statistics showed that MS-EPI is better than SS-EPI. Though SS-EPI requires only a single radio frequency excitation pulse, and it is most widely used DWI technique in clinical practice but it was known to be very susceptible to main field in-homogeneity, which may lead to severe image degradation.

$>$ The limitation with the study was that the apparent diffusion coefficient was not evaluated to confirm decreased diffusion and contrast T1-weighted imaging was not evaluated. Here the authors only focused on evaluating the 2 echo planar methods, and MS-EPI showed positive results in this study.

\section{Sam Khemani et al.:-}

In this paper, researchers had reviewed the imaging techniques used for postoperative middle ear cholesteatoma. They had reviewed CT (HRCT), delayed contrast MRI, diffusion weighted MRI (echo planar and non echo planar). Also, the current role of imaging that had shown the greatest promise in detecting cholesteatoma following surgery was also reviewed.

\section{Critical Remarks:-}

$>$ Though HRCT showed signs of residual cholesteatoma but it was unreliable in differentiating residual cholesteatoma from inflammation, fibrosis, and granulation cholesteatoma granuloma in the middle ear cleft. Though delayed contrast MRI has been reported to detect cholesteatoma greater than $3 \mathrm{~mm}$ in size.

$>$ Currently non EPI DWI is most popular imaging techniques for residual cholesteatoma in comparison to other imaging techniques, but the main concern with this technique is its inability to detect cholesteatoma pearls less than $3 \mathrm{~mm}$.

[

E Flook, S Izzat et al.:-

In this paper authors had presented a modified version of echo planar magnetic resonance. They had done some software adjustment in the non-echo planar magnetic imaging techniques by modifying the technique's parameters.

The main aim of modifying the parameters of non-echo planar magnetic resonance imaging by the authors was to detect the cholesteatoma as reliably and effectively as non-echo planar diffusion weighted magnetic resonance imaging. Seven patients were investigated for primary cholesteatoma, four of these patients' scans showed cholesteatoma while three were negative for cholesteatoma. Eight patients were investigated for recurrent or residual cholesteatoma; five of these patients' scans were positive for cholesteatoma while three were negative. All scans report co-related with clinical/surgical findings.

\section{Critical Remarks:-}

With all the results and conclusion, it was found that the new modified techniques is as reliable and effect as non echo planar diffusion weighted MRI. The study also suggests that it can detect cholesteatoma as small as $4 \mathrm{~mm}$ and detection of smaller cholesteatoma was also possible. 


\section{Sherif Abdel Fattah Khedr et al.:-}

The aim of this study was to determine the role of diffusion weighted MR imaging for the detection of cholesteatoma in patients who had undergone middle ear surgery (Tympanoplasty). 21 patients were taken as study and referred for MRI using DW fast spin-echo sequence, T2-weighted spin-echo sequence and T1 weighted before and after contrast injection as well as delayed contrast-enhanced. The recent MRI sequences showed the presence of cholesteatoma in 11 ears only, whereas surgically proven recurrent or residual cholesteatoma was found in 13 ears.

\section{Critical remarks:-}

DW MRI combined with MR imaging accurately depicted 11 of 13 cholesteatoma giving $84.6 \%$ sensitivity. All the MR imaging's patients without cholesteatoma was correctly interpreted as showing negative findings for cholesteatoma giving, specificity $100 \%$.

\section{Bee See Goh et.al:-}

This proposed paper is a retrospective review of congenital cholesteatoma that was managed surgically. There were 5 cases out of which 3 had intact tympanic membrane and 2 had perforation at the anterior superior quadrant. 4 cases had extensive ossicular erosion with preoperative hearing worse than $40 \mathrm{db}, 4$ cases underwent canal wall down mastoid surgery and 1 went canal wall up surgery.

\section{Critical Remark:-}

Congenital cholesteatoma is a rare disease with most of its cases leading to hearing loss and other consequences, the treatment for this disease is surgery i.e. canal wall down surgery is recommended, whose goal is complete removal of disease with the restoration of hearing if possible.

\section{P A Clark et al.:-}

In this proposed paper the authors' had shared their views related to MRI used for the treatment detection of residual cholesteatoma. In this paper it was discussed, "were MRI techniques were not good enough for the detection of residual cholesteatoma and was it necessary to go for second look surgery".

Researchers had discussed various techniques of magnetic resonance imaging in the paper, such as single shot turbo spin diffusion weighted, late post gadolinium T1 weighted, propeller diffusion weighted.

\section{Critical Remarks:-}

$>$ One criticism of the diffusion weighted image technique is its inability to clearly show the body anatomical features of its temporal bone. This makes precise localization of any signals suggesting cholesteatoma somewhat difficult.

$>$ Only one case study is discussed in the paper and demonstrates both the success and failure of these techniques.

\section{Mudit Jindal et al.:-}

Researchers' aim was to study the usefulness of diffusion weighted echo-planar imaging (DW-EPI) for the diagnosis of residual cholesteatoma .50 patients were taken for case study and divided into two group. In the first preoperative group, 15 patients were taken and DW-EPI identified correctly 15 cases of cholesteatoma, no false positive or false negative findings.

In the second group, DW-EPI was performed after mastoid surgery in 35 patients. DW-EPI correctly identified cholesteatoma in 29 out of 35 patients. Residual cholesteatoma was correctly identified in 15 out of 18 patients and tiny residual pearls $(2-5 \mathrm{~mm})$ were missed in 3 patients.

\section{Critical Remarks:-}

$>$ DW-EPI is unable to detect tiny residual pearls of cholesteatoma, which are less than $5 \mathrm{~mm}$. This appears to be biggest disadvantage.

$>$ DW-EPI is more prone to artifact at the air- bone and air -tissue interfaces and can give rise to distorted image and false positive signals. Even the author's study three patients were misinterpreted as cholesteatoma.

$>$ In this study, DW-EPI has been seen to have a sensitivity of $83 \%$ in detecting residual cholesteatoma. 


\section{Nuri Cagatay Cimsit et al.:-}

The aim of this study was to evaluate that the diffusion weighted MR imaging alone can be reliable alternative to CT, without use of contrast agent for follow-up of post-operative patients in detecting recurrent cholesteatoma. Case studies of 26 patients with mastoidectomy reporting for routine follow-up CT after mastoidectomy were taken. With 26 patients examined, 14 had no high signal intensity on DWI and the loss of aeration in the middle ear was not evaluated as recurrent cholesteatoma. I $t$ was confirmed after surgery, leading to negative predictive value $100 \% .12$ patients had high signal intensity on DWI in at least a portion of the loss of aeration on T2W images, 11 of these patients' surgical diagnosis was recurrent cholesteatoma. Thus the positive predictive value was $91.7 \%$.

\section{Critical Remarks:-}

$>$ Though with all the case studies, it had been found that DWI MR imaging is quite promising in finding recurrent cholesteatoma .But here the researchers had used echo planar based DWI, which is prone to susceptibility artifacts originating from air-bone interfaces and has low spatial resolution characteristics.

$>$ Also, one false positive case is also found in the study .Apart from all these there were problems with the detection of cholesteatoma based on their size. Cholesteatoma tissue less than $5 \mathrm{~mm}$ in size was hard to detect.

\section{Bert De Foer, Jean-Phillippe Vercruysse et al:-}

In this paper the researchers aim was to compare non-echo planar (Non-EP) diffusion weighted (DW) imaging, delayed gadolinium- enhanced T1 weighted magnetic resonance imaging, and also the combination of both technique for the evaluation of patients with cholesteatoma. To measure the accuracy of all three techniques their sensitivity, specificity, positive predictive value and negative predictive values were used.

This paper consists of total 120 cases of cholesteatoma, divided in to three datasets. After applying all the three techniques, results were compared with surgical results, regarding standard of reference. Results showed that nonecho planar (EP) diffusion weighted (DW) imaging had significantly higher sensitivity than delayed gadoliniumenhanced T1 weighted MR imaging. The combination of delayed gadolinium-enhanced T1 weighted MR imaging and non eco planar diffusion weighted imaging yielded no higher sensitivity than non echo planar diffusion weighted imaging alone.

\section{Critical Remarks:-}

With all the comparisons made and the result obtained it was suggested that even non experienced reader were able to correctly diagnosis the presence of a cholesteatoma with the help of the non echo planar diffusion weighted imaging sequences. On the basis of result it was stated that delayed gadolinium enhanced T1 weighted sequences are no longer used.

\section{Lela Migirov et al.:-}

In this paper the researchers had supported the use of magnetic resonance imaging as more suitable than CT in postoperative follow-up protocols for cholesteatoma. Supporting MRI over CT was because of their experience with the meningoceles in patients who had undergone mastoidectomy for cholesteatoma in the past and who then underwent what turned out to be unnecessary second-look procedures due to misdiagnosis based on CT findings.

In this study researchers have used the EPI DWI version of MR imaging. 19 patients were taken as study that demonstrated one false positive and six true positive interpretations of images. The MRI diagnosis of cholesteatoma was confirmed at surgery in each of the six true positive interpretations.

\section{Critical Remarks:-}

$>$ Though MRI was definitely more suitable in comparison to CT, with the technique reducing the rate of secondlook surgeries from $50-60 \%$ of cholesteatoma cases to $10 \%$.

But here researchers had only studied one version of MRI i.e. EPI DWI (Echo Planar Imaging-Diffusion Weighted Imaging) which had some drawbacks such as, artifacts from air bone tissue interfaces and detecting the cholesteatoma of minimum certain size only. 2) Researchers had not included the Non Echo Planar Diffusion Weighted Imaging, which is much better than EPI DWI and had no drawbacks of the previous one. 
[23] J. Ph. Vercruysse. et al.:- In this proposed paper researchers had reviewed the value and limitation of new MRI techniques used in pre and post operative MRI of cholesteatoma. They had reviewed delayed post gadolinium magnetic resonance imaging and diffusion weighted echo planar versus non echo planar DWI.

Delayed post gadolinium magnetic resonance imaging is used for differentiating non-enhancing and avascular cholesteatoma from slow enhancing inflammatory and/or scar tissue. It was able to detect post-operative residual cholesteatoma pearls as small as $3 \mathrm{~mm}$.

But in echo planar MR imaging, the major disadvantage was the presence of important susceptibility artifacts at the air-bone of the skull, the variable distortion of the images, and the low spatial resolution. To overcome all these disadvantages of echo planar MR imaging, non echo planar MR imaging was introduced.

\section{Critical Remarks:-}

After reviewing both the techniques, it was found that for detecting pre- operative primary acquired and congenital cholesteatoma and for the postoperative residual or recurrent cholesteatoma, consists of combination of both techniques. It was stated that, MRI including non EPI-DW and late post gadolinium T1 weighted images, should be used rather than $\mathrm{CT}$ to evaluate cholesteatoma before second look surgery

\section{Bert de Foer, Filip Deckers et al:-}

The main aim of this study was to evaluate the efficiency of non echo planar diffusion weighted magnetic resonance imaging in the detection of postoperative (canal wall-up mastoidectomy) residual cholesteatoma. 32 patients were taken in the study and were investigated with MRI, including late post gadolinium T1-weighted sequence and non EPI-DWI sequences. Of the 19 patients who underwent surgeries, 18 were proven to have correct diagnosis on the basis of the single shot turbo spin diffusion weighted sequence and there were true positive findings in 9 patients for cholesteatoma.

\section{Critical Remarks: -}

$>$ With the case study in the paper it showed that non echo planar succeeded in demonstrating middle ear cholesteatoma as small as $2 \mathrm{~mm}$ in non operated ears, whereas late post gadolinium T-1 weighted sequence s ale to detect residual cholesteatoma only as small as $3 \mathrm{~mm}$.

$>$ In the end they have concluded that non echo planar diffusion weighted is far superior in the detection of residual cholesteatoma.

\section{B. De Foer et al.:-}

This paper had proposed the comparison between two techniques used for the diagnoses of middle ear cholesteatoma, i.e. single shot turbo spin echo DWI versus EPI-DWI.

With the advancement in MRI techniques in detecting cholesteatoma, EPI-DWI was quite an improvement over the other imaging techniques, but still EPI-DWI suffers with the numerous artifacts such as eddy current artifact, susceptibility artifacts, chemical shift, and motion artifacts. Turbo spin echo (TSE) DWI MR is known to lack significant image distortions and other artifacts.

In this paper, a case study was done where the diagnosis of cholesteatoma was done on the basis of the late post gadolinium T1 weighted images and single shot TSE-DWI and surgery confirmed a 5mm size cholesteatoma lesion.

\section{Critical Remarks:-}

$>$ The case study that was taken in this paper proved to be quite successful, with the help of late post gadolinium T1 weighted images and single shot TSE DWI, it became easy for diagnosis of a primary acquired cholesteatoma.

$>$ Though further studies on larger series are needed to prove the value of a single shot TSE DWI in combination with late post gadolinium T1- weighted images for the diagnosis of primary acquired and residual cholesteatoma.

Jean-Philippe Vercruysse et al.:-

Researchers' purpose of this study was to evaluate the value of echo-planar diffusion weighted MR imaging (EPDWI) using $3 \mathrm{~mm}$ thick slices in detecting primary or residual cholesteatoma in a large series of surgically verified 100 cases. In the first group of 55 patients (for primary cholesteatoma), correct diagnosis was found in $84 \%$. False 
negatives was found in 9 patients, also the minimum largest lesion size found was $5 \mathrm{~mm}$. whereas in second group consisting of 45 patients, residual cholesteatoma was found in 7 patients only. A correct diagnosis using EP-DWI was made only in one patient with residual pearl with shortest diameter of $6 \mathrm{~mm}$. No false negatives were found in second group.

\section{Critical Remarks:-}

$>$ From above diagnosis, it was found that the value of EP-DWI in detecting the primary cholesteatoma was better, but shows the poor capability in detecting small residual cholesteatoma.

$>$ Also major limitation for detecting cholesteatoma in diffusion weighted sequences is definitely the size of the lesion (4-5mm), combined with the low spatial resolution, the relative thick slices and the air-bone artifacts.

\section{Frederique Dubrulle et al..:-}

The main aim of this paper was to evaluate a fast spin echo diffusion weighted sequence for magnetic resonance imaging of recurrent cholesteatoma for patients who had undergone middle ear surgery. 24 consecutive patients were taken for study that had undergone either a canal wall-up or wall-down mastoidectomy, their MRIs were taken and compared with surgical results. For statistical analysis sensitivity, specificity, and positive and negative predictive values were evaluated.

In 10 out of 24 patients, no recurrence of cholesteatoma was diagnosed at MR imaging and no recurrence cholesteatoma was found at surgery. In 14 patients, recurrence cholesteatoma was diagnosed at MR imaging and in comparison with surgical result, there was one false positive finding. As a result the positive predictive value was $93 \%$.

\section{Critical Remarks:-}

$>$ After the case studies, the result showed that fast spin echo diffusion weighted MRI is quite reliable, it has shown no false negative value.

$>$ But it has some limitation like it appeared that it has few artifacts at the air-bone interface, image distortions along the phase-encoding directions were unavailable. Analyzing the lesions smaller than particular size was hard to detect.

$>$ Further investigation were necessary to determine whether the diffusion weighted fast spin echo sequence should become a routine evaluation in MRI to differentiate postoperative granulation tissue from recurrent cholesteatoma without the use of delayed contrast enhanced T1 weighted imaging.

\section{Marc T. Williams and Denis Ayache:-}

The main aim of this paper was to present the principles and he indications of surgical treatment of middle ear pathologies and to review the imaging findings after middle ear surgery. In this review paper it was strictly focused on the common middle ear disease like chronic otitis media, cholesteatoma, otosclerosis etc and imaging technique that were used for these diseases.

Paper consists of imaging techniques like computed tomography, MR imaging (all versions) and their advantages and limitations. Whereas, also the paper contain about the middle ear disease such as chronic otitis media, its potential of destruction, kind of imaging and surgery technique needed. Apart from this they had also talked about otosclerosis disease.

\section{Critical Remarks:-}

The paper doesn't much contain the imaging techniques and their uses. Their main focus was on the middle ear diseases.

\section{Marta E. Heilbrun, Karen L. Salzman et al.:-}

The main purpose of this study was to define the imaging features of External Auditory Canal Cholesteatoma (EACC) at Computed Tomography also in addition they try to determine those features that distinguish EACC from its clinical differential diagnosis. 13 cases of EAC cholesteatoma were reviewed and CT imaging in all 13 cases showed a soft tissue with adjacent bone erosion. 


\section{Critical Remarks:-}

Case studies showed that imaging can be valuable in evaluation of EACC. However, the CT appearance of EACC is not well defined in any literature. With high resolution temporal bone CT examination, EACC is most commonly seen as an EAC soft tissue mass with associated bone erosion and intramural bone fragment.

Marc T. Williams et al.:- Here the researchers' objective were to determine if MR imaging, using delayed contrast -enhanced T1 weighted images, was able to detect postoperative residual cholesteatoma in patients who have undergone canal wall-up Tympanoplasty .Case study was done on 18 patients and results were separately compared with surgical second- look findings. Sensitivity, specificity and predictive values were evaluated for early and delayed post-contrast MR imaging, compared with second look surgery. Result shows that residual cholesteatoma was found in 9 of 18 patients at revision surgery. Cholesteatoma was correctly found in 8 cases on delayed contrastenhanced $\mathrm{T} 1$ weighted images.

\section{Critical Remarks:-}

$>$ The size of the smallest residual cholesteatoma detected with the delayed contrast - enhanced MR technique was $2.5 \mathrm{~mm}$ only. Indicating that smaller lesions may be missed or confused with granulomatous tissue.

$>13.3 \%$ cases were found false -negative due to small size of lesions. Therefore, further MR imaging can be required at initial findings.

\section{Sharad Maheshwari et al.:-}

In this paper, researchers had proposed that diffusion weighted imaging can be used for differentiating recurrent cholesteatoma from granulation tissue after Mastoidectomy. In this paper only one case study is taken and the results showed that cholesteatoma was bright on diffusion weighted images.

\section{Critical Remarks:-}

The case study showed that diffusion-weighted imaging may be helpful in identifying recurrent cholesteatoma. Diffusion weighted imaging had been accepted as an curate method for detecting acute stoke and is also being evaluated in variety of other intracranial disease process.

\section{Biodun Ogungbo et al:-}

This proposed paper represents a case report of Co-existing cholesteatoma and vestibular schwannoma in the right as well as on the left mastoid of a 69 year old man . Magnetic resonance imaging(MRI) and MR cerebral angiography was carried on along with CT scans. These revealed a large $(4.5 * 3 * 2.5 \mathrm{~cm})$ lobulated lesion within the petrous and occipital bones on the right side and replacing almost of the right mastoid. CT scan demonstrated erosion of the right temporal bone, the posterior wall of which was completely destroyed.

\section{Critical Remark:-}

An open biopsy of the right sided mastoid lesion was undertaken. The operation was performed through a small retro-auricular incision over the soft tissue of the bony destruction. The patient has a functional hearing in both the ears, slightly worse on the left.

\section{Conclusion and Future scope:-}

Reviews on various techniques have been done in order to identify and classify the middle ear cholesteatoma. Various techniques like Computed Tomography (CT), Echo-planar Diffusion Weighted Magnetic Resonance Imaging. Non echo- planar Diffusion Weighted Magnetic Resonance Imaging had been used. Limitations and advantages of these techniques are reviewed. The main aim of this review paper was to identify and detect Middle Ear Cholesteatoma .We has observed there are various techniques that are very useful, and detect Cholesteatoma with at most 94-95\% accuracy. Apart from that each technique has their own accuracy in finding out the particular diseases. Also it has been observed that these techniques can be used in detecting, identifying various other diseases apart from the disease for that it has been proposed.

\section{Acknowledgement:-}

This research paper was supported by Prof. G.N. Purohit Dean, AIM \& ACT, Banasthali University, for providing the opportunity to carry out our 10 months M.tech. Internship program. 
We would also like to show our gratitude to Dr. Pawan Singhal, Department of ENT \& Head Neck Surgery (SMS Medical College \& Hospital, Jaipur) for sharing their pearls of wisdom with us during the course of this research.

\section{References:-}

1. Mihael Ries, Mirjana Kosti', Jakov Ajduk, Robert Troti', Vladimir Bedekovi', Brazilian Journal of OTORHINOLARYNGOLOGY,ELSEVIER, http://dx.doi.org/10.1016/j.bjorl.2015.09.003 , Received 31 July 2015; accepted 29 September 2015, Braz J Otorhinolaryngol. 2016.

2. Filippo Di Lella, Andrea Bacciu, Enrico Pasanisi, Marco Ruberto, Giulia D'Angelo, Vincenzo Vincenti,Clinical findings and surgical results of middle ear cholesteatoma behind an intact tympanic membrane in adults, Research gate, May 2016.

3. Andrea Elefante,Michele Cavaliere,C. Russo,G. Caliendo, Mariano Marseglia,Domenico Cicala,D. Piccolo,Maurizio Iengo,Antonella Miriam Di Lullo,L. Brunetti, A. Palma, Arturo Brunetti, Diffusion Weighted MR Imaging of Primary and Recurrent Middle Ear Cholesteatoma: An Assessment by Readers with Different Expertise, Research Gate, DOI: 10.1155/2015/597896,February 2015.

4. Robert Nash,Phui Yee Wong,Ali Kalan,Ravi K lingam,Arvind Singh, Comparing Diffusion Weighted MRI in the detection of Post-Operative Middle Ear Cholesteatoma in Children and Adults, Research gate, DOI:10.1016/j.ijporl.2015.10.025, November 2015.

5. Ahmed Abdel Razek, Mohamed Rashad Ghonim, Bassem Ashraf, Computed Tomography Staging of Middle Ear Cholesteatoma, research gate, DOI: 10.12659/PJR.894155 - Source: PubMed, July 2015.

6. S VELTHUIS, K J VAN EVERDINGEN, J J QUAK, D R COLNOT, The value of non echo planar, diffusionweighted magnetic resonance imaging for the detection of residual or recurrent middle-ear cholesteatoma, The Journal of Laryngology \& Otology (2014), 128, 599-603, doi:10.1017/S0022215114001418.

7. Sang Hyuk Ahn, Yong-Woo Kim, Seung Kug Baik, Jae-Yeon Hwang, Il-Woo Lee, Usefulness of Computed Tomography Hounsfield Unit Measurement for Diagnosis of Congenital Cholesteatoma1Original Article; pISSN 1738-2637 / eISSN 2288-2928; J Korean Soc Radiol 2014;70(2):153-158.

8. Ana Flávia Assis de Ávila, Bruna de Oliveira Melim Aburjeli, Wanderval Moreira, Emília Guerra,Pinto Coelho Motta, Marcelo Almeida Ribeiro, Renata Lopes Furletti Caldeira Diniz, Imaging evaluation of middle ear cholesteatoma: iconographic essay, Radiol Bras. 2013 Jul/Ago;46(4):247-251; 0100-3984 @ Colégio Brasileiro de Radiologia e Diagnóstico por Imagem.

9. Maged B Naguib, Surgical Management of Cholesteatoma Growing Medially into the Petrous part of the Temporal Bone, ISSN: 2161-1076 SCR, an open access journal.

10. Carleton Eduardo Corrales,Nikolas H Blevins, Imaging for evaluation of cholesteatoma: Current concepts and future directions, Research Gate, 2013.

11. Lauren L. Levy, Nancy Jiang, Eric Smouha, Rebecca Richards-Kortum,Andrew G. Sikora, Optical Imaging with a High Resolution Micro endoscope to Identify Cholesteatoma of the Middle Ear, NIH Public Access, 2013.

12. Dalia Monir Fahmy, Sameh M. Ragab, Detection of post operative residual cholesteatoma using PROPELLER DWI combined with conventional MRI, The Egyptian Journal of Radiology and Nuclear Medicine (2012) 43, 543-548.

13. K. Yamashita,T. Yoshiura,A. Hiwatashi,H. Kamano,T. Dashjamts,S. Shibata,A. Tamae,H. Honda, Detection of Middle Ear Cholesteatoma by Diffusion-Weighted MR Imaging: Multishot Echo-Planar Imaging Compared with Single-Shot Echo-Planar Imaging, AJNR Am J Neuroradiol 32:1915-918 Nov 2011 www.ajnr.org.

14. Sam Khemani, Arvind Singh, Ravi K Lingam,A Kalan, Imaging of postoperative middle ear cholesteatoma, DOI: 10.1016/j.crad.2010.12.019 - Source: PubMed, Research gate ,April 2011.

15. E FLOOK, S IZZAT, A ISMAIL, Cholesteatoma imaging using modified echo-planar diffusion-weighted magnetic resonance imaging, The Journal of Laryngology \& Otology (2011), 125, 10-12.JLO (1984) Limited, 2010; doi:10.1017/S0022215110001805.

16. Sherif Abdel Fattah Khedr, Ahmed Mohamed Adly, Ahmed Fathi ,Lobna El Fiky, Amgad Obaid, Role of diffusion-weighted MRI in the detection of cholesteatoma after Tympanoplasty, Egyptian Journal of Ear, Nose, Throat and Allied Sciences (2011) 12, 141-148.

17. Bee See Goh,A R Faizah,Husain Salina, Abdullah Asma,L Saim, Congenital cholesteatoma: Delayed diagnosis and its consequences, research gate, September 2010.

18. M P A CLARK, B DWESTERBERG, D M FENTON, The ongoing dilemma of residual cholesteatoma detection: are current magnetic resonance imaging techniques good enough? The Journal of Laryngology \& Otology (2010), 124, 1300-1304. JLO (1984) Limited, 2010. 
19. Mudit Jindal, Jayesh Doshi , Manoj Srivastav ,David Wilcock, Richard Irving, Ranit De, Diffusion-weighted magnetic resonance imaging in the management of cholesteatoma, Eur Arch Otorhinolaryngol (2010) 267:181185 DOI 10.1007/s00405-009-1023-7.

20. Nuri Cagatay Cimsita, Canan Cimsitc, Begumhan Baysal ,Ilteris Cagatay Ruhie,Suha Ozbilgene, Elif Ayanoglu Aksoyg, Diffusion-weighted MR imaging in postoperative follow-up: Reliability for detection of recurrent cholesteatoma, European Journal of Radiology 74 (2010) 121-123;ELSEVEIR.

21. Bert De Foer, Jean-Philippe Vercruysse ,Anja Bernaerts, Joke Meersschaert, Christoph Kenis ,Marc Pouillon , Luc De Beuckeleer, Johan Michiels, Kris Bogaerts, Filip Deckers, Thomas Somers, Robert Hermans , Erwin Offeciers , Jan W. Casselman , Middle Ear Cholesteatoma: Non-Echo-planar Diffusion-weighted MR Imaging versus Delayed Gadolinium-enhanced T1-weighted MR Imaging_Value in Detection, radiology.rsna.org ; Radiology: Volume 255: Number 3-June 2010.

22. Lela Migirov ,Sigal Tal, Ana Eyal , Jona Kronenberg, MRI, not CT, to rule out recurrent cholesteatoma and avoid unnecessary second-look mastoidectomy, IMAJ・VOL 11• MARCH 2009.

23. J. Ph. Vercruysse, B. De Foer, Th. Somers, J. Casselman, E. Offeciers, Magnetic resonance imaging of cholesteatoma: an update, B-ENT, 2009, 5, 233-240.

24. Bert De Foer,jean-Philippe Vercruysse,Anja Bernaerts,Filip Deckers, Marc Pouillon,Thomas Somers,Jan Casselman ,Erwin Offeciers, Detection of postoperative residual cholesteatoma with non-echo-planar diffusionweighted magnetic resonance imaging, Research gate June 2008, DOI: 10.1097/MAO.0b013e31816c7c3b • Source: PubMed.

25. B. De Foer,J.-P. Vercruysse,B. Pilet,J. Michiels,R. Vertriest,M. Pouillon,T. Somers,J.W. Casselman,E. Offeciers, Single-Shot, Turbo Spin-Echo, Diffusion-Weighted Imaging versus Spin-Echo-Planar, DiffusionWeighted Imaging in the Detection of Acquired Middle Ear Cholesteatoma, www.ajnr.org.

26. Jean-Philippe Vercruysse,Bert De Foer,Marc Pouillon,Thomas Somers,Jan Casselman,Erwin Offeciers, The value of diffusion-weighted MR imaging in the diagnosis of primary acquired and residual cholesteatoma: a surgical verified study of 100 patients, Eur Radiol (2006) 16: 1461-1467 DOI 10.1007/s00330-006-0160-2.

27. Frederique Dubrulle,Raphaelle Souillard,Davida Chechin, Franc, ois M. Vaneecloo,Alain Desaulty,Christophe Vincent, Diffusion-weighted MR Imaging Sequence in the Detection of Postoperative Recurrent Cholesteatoma, Radiology: Volume 238: Number 2-February 2006.

28. Marc T. Williams, Denis Ayache, Imaging of the postoperative middle ear, Eur Radiol (2004) 14:482-495 DOI 10.1007/s00330-003-2198-8.

29. Marta E. Heilbrun, Karen L. Salzman, Christine M. Glastonbury, H. Ric Harnsberger, Richard J. Kennedy, and Clough Shelton, External Auditory Canal Cholesteatoma: Clinical and Imaging Spectrum, AJNRa Am J Neuroradiol 24:751-756, April 2003.

30. Marc T. Williams,Denis Ayache,Corinne Alberti,Françoise Héran,François Lafitte, Monique ElmalehBergès,Jean-Daniel Piekarski, Detection of postoperative residual cholesteatoma with delayed contrastenhanced MR imaging: initial findings, Eur Radiol (2003) 13:169-174 DOI 10.1007/s00330-002-1423-1.

31. Sharad Maheshwari and Suresh K. Mukherji, Diffusion-Weighted Imaging for Differentiating Recurrent Cholesteatoma from Granulation Tissue after Mastoidectomy: Case Report, AJNR Am J Neuroradiol 23:847849, May 2002.

32. Biodun Ogungbo, Damian Holliman, A. David Mendelow, John Hill, Co-existing cholesteatoma and vestibular schwannoma, The Journal of Laryngology \& Otology June 2002, Vol. 116, pp. 460-463. 